\title{
The preparation of Fe-Co double nanoparticle magnetic fluid by pulse laser deposition
}

\author{
Zheng.Qi.Lian", Li.Gao², Gui.Fu. Dong ${ }^{1, *}$ \\ 1. Dalian University, Dalian 116622, China; \\ 2. College of Engineering Science and Technology, Shanghai Ocean University, shanghai, 201306, \\ China \\ E-mail address: dgfu0451@sina.com,
}

\begin{abstract}
Keywords: Pulse laser deposition; Double nanoparticle magnetic fluid; Dispersibility ; Magnetization saturation
\end{abstract}

\begin{abstract}
The Fe-Co double nanoparticle magnetic fluid prepared by pulse laser deposition was investigated. The results show that peak height of magnetic fluid under 5000 Gs magnetic fluid gradually reduced with increase mass ratio of $\mathrm{Fe}(\mathrm{CO})_{5}$ and $\mathrm{Co}_{2}(\mathrm{CO})_{8}$, and peak height is biggest when mass ratio of $\mathrm{Fe}(\mathrm{CO})_{5}$ and $\mathrm{Co}_{2}(\mathrm{CO})_{8}$ is $5: 1$. It is can seen that magnetic fluid consist of $\mathrm{Fe}_{3} \mathrm{O}_{4}$ and $\mathrm{CoFe}_{2} \mathrm{O}_{4}$ particle. The particles uniform covered surfactant in magnetic fluid and favorable distribution with most particle size is approximate $14 \mathrm{~nm}$. The magnetic fluid is super paramagnetic functional materials with magnetic saturation of the $0.025 \mathrm{~T}$. The friction coefficient of magnetic fluid rapidly decreases than paraffin oil with increase of rotational speed and friction time lubricated.
\end{abstract}

\section{Introduction}

Magnetic fluid is kinds of stable colloid system consist of magnetic nanoparticles dispersed and suitable liquid carrier. The magnetic fluid was first synthesized in early 1960. Magnetic fluid is stable colloidal suspensions consisting of single-domain magnetic particles coated with a surfactant and immersed in a carrier fluid. They possess many exclusive physical properties [1-8]. The stability of magnetic fluid depends upon a balance between repulsive and attractive interactions among nanoparticles [9]. Besides the thermal motion, the steric and electrostatic repulsive interactions are against Van der Waals and dipolar attractive interactions. The material exhibits the combination of both normal liquid behavior and magnetic controllable properties. This intelligent material has attracted much attention due to its potential applications in aviation, apparatus, industrial and biological fields, etc. The magnetic properties of magnetic fluid depend strongly on the species of the particles and the concentration of the magnetic material. In the presence of a magnetic field, the magnetic moment of the particles will try to align with the magnetic field direction leading to a macroscopic magnetization of the fluid. When the external field is removed, the particles quickly randomize the directions of their magnetic moment and the fluid loses its magnetism. However, the magnetic properties of iron oxide-based magnetic fluid are not sufficient for a number of purposes. So, we prepared $\mathrm{Fe}-\mathrm{Co}$ double nanoparticle magnetic fluid in order to increase magnetic properties. In addition, by the vast number of scholars study found that, by adopting the method of chemical preparation of $\mathrm{Co}-\mathrm{Fe}_{3} \mathrm{O}_{4}$ magnetic lubricating oil has good stability, high saturation magnetization[10-14].It's widely used in industrial sliding bearing, mineral separation, sealing, damping shock, precision grinding, transducer and heat engineering areas[14-18].

But the preparation of magnetic fluid is quite complicated and time-consuming. A variety of techniques have been developed to prepare magnetic fluid using metal particles such as spark erosion and vacuum evaporation [19]. One of the major difficulties encountered is that the magnetization of the metal particles decays with time, due to the lack of oxidization resistance in the ambient

\footnotetext{
${ }^{*}$ Corresponding Author: Tel.: +86 41187402423.

E-mail address: dgfu0451@sina.com,
} 
environment. Making use of the high efficiency and rapidity of plasma technique, a large number of electrons, ions, excited atoms and molecules, and activated species such as radicals were obtained in the reactive device for preparations of nano-magnetic particles. These active particles can make many impossible or difficult reactions to take place or quickly take place; new materials can be made with pre-designed model. Therefore it is a very effective method in molecular activation. By using the pulse laser deposition method, we have prepared magnetic fluid in about $2 \mathrm{~h}$, reducing the processing time to $80 \%$.

In this article, we have made an effort to the synthesis of magnetic fluids with extraordinary magnetic properties by pulse laser deposition method for the first time. In the magnetic fluids, the nanosized Fe-Co powders coated by surfactants were dispersed in carrier liquid. The assays performed for the preparation, morphology, structure and magnetic property, are discussed in the following sections.

\section{Experimental}

The Fe-Co double nanoparticle magnetic fluid were prepared with different mass ratio of $\mathrm{Fe}(\mathrm{CO})_{5}$ and $\mathrm{Co}_{2}(\mathrm{CO})_{8}$ by pulse laser deposition method in frequency $4 \mathrm{HZ}$ and substrate temperature is $200{ }^{\circ} \mathrm{C}$. In a first step, In order to favorable distribution different mass ratio of $\mathrm{Fe}(\mathrm{CO})_{5}$ and $\mathrm{Co}_{2}(\mathrm{CO})_{8}$ by ultrasonic method. Secondly, the primary surfactant (oil) takes in reaction vessel and dispersed in the $\mathrm{Fe}(\mathrm{CO})_{5}$ and $\mathrm{Co}_{2}(\mathrm{CO})_{8}$. Lastly, reaction vessel put in PLD-450 vacuum system with system evacuated to $10^{-4} \mathrm{~Pa}$ and heated at $200{ }^{\circ} \mathrm{C}$. So that the Fe-Co double nanoparticle magnetic fluid can be produced under the condition of the plasma and temperature field. Then the Fe-Co double nanoparticles coated with oil was washed four times with distilled water so as to remove impurity. Then water in the particles was removed by washing with acetone. The resulting particles coated with a secondary surfactant were dispersed in a-olefinic hydrocarbon synthetic oil (PAO4) by ultrasonic method. The final fluid was centrifuged at $12,000 \mathrm{rpm}$ for $10 \mathrm{~min}$.

To measure particle size and morphology of Fe-Co double nanoparticle magnetic fluid we use JEM-2000EX TEM. Its amplification factor is $200 \mathrm{~K}$. And X-ray diffraction (XRD) was used to determine the phase structure. Saturation magnetization measurements were taken using the physical property measurement system (Quantum Design) in an applied field up to $5 \mathrm{~T}$. The MRH-3 four-ball machine was used to test the frictional properties of $\mathrm{Fe}-\mathrm{Co}$ double nanoparticle magnetic fluid compared with paraffin oil and so on. Its working principle is simple that the friction pair components , the armillary component (the material of 20CrNiMo, the size of $\Phi 49.22 \mathrm{~mm} \times 13.06 \mathrm{~mm}$ ) and the square test component (the material of $45 \#$ steel, the size of $12.32 \mathrm{~mm} \times 12.32 \mathrm{~mm} \times 19.05 \mathrm{~mm}$ ) will friction under different lubricants in a certain load. The experimental temperature is $25{ }^{\circ} \mathrm{C}$. The test time of each sample is 10 minutes. The rotating speed of the armillary component is $600 \mathrm{r} / \mathrm{min}$.

\section{Results and Discussion}

The peak height of the Fe-Co double nanoparticle ferrofluids sample with is presented in Fig. 1. From the figure we can see that the peak height of magnetic fluid under 5000 Gs magnetic fluid gradually decrease with increase mass ratio of $\mathrm{Fe}(\mathrm{CO})_{5}$ and $\mathrm{Co}_{2}(\mathrm{CO})_{8}$. The biggest peak height observed when mass ratio of $\mathrm{Fe}(\mathrm{CO})_{5}$ and $\mathrm{Co}_{2}(\mathrm{CO})_{8}$ is $5: 1$. That is to say, the may obtained higher quality when mass ratio of $\mathrm{Fe}(\mathrm{CO})_{5}$ and $\mathrm{Co}_{2}(\mathrm{CO})_{8}$ is $5: 1$.

Fig. 2 show the X-ray diffraction pattern of the Fe-Co double nanoparticle when mass ratio of $\mathrm{Fe}(\mathrm{CO})_{5}$ and $\mathrm{Co}_{2}(\mathrm{CO})_{8}$ is 5:1. It was indicated that the synthesized samples consisted of $\mathrm{Fe}_{3} \mathrm{O}_{4}$ and $\mathrm{CoFe}_{2} \mathrm{O}_{4}$ particles. So the main content of the complex Fe-Co double nanoparticle magnetic fluid are $\mathrm{Fe}_{3} \mathrm{O}_{4}$ and $\mathrm{CoFe}_{2} \mathrm{O}_{4}$. The broadening Bragg peaks for the as-prepared $\mathrm{Fe}_{3} \mathrm{O}_{4}$ and $\mathrm{CoFe}_{2} \mathrm{O}_{4}$ are due to their small particle size. 


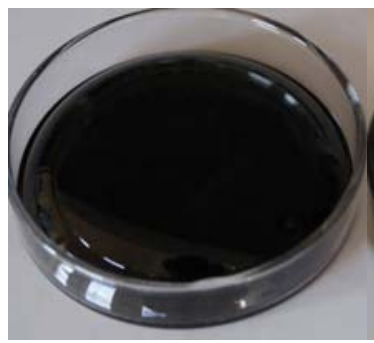

(a)

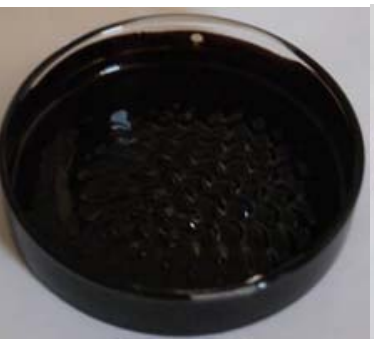

(b)

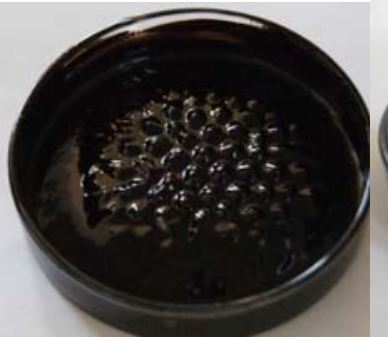

(c)

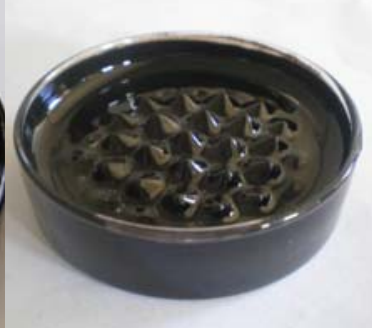

(d)

(a) $\mathrm{m}\left(\mathrm{Fe}(\mathrm{CO})_{5}\right): \mathrm{m}\left(\mathrm{Co}(\mathrm{CO})_{8}\right)=8: 1$

(b) $\mathrm{m}\left(\mathrm{Fe}(\mathrm{CO})_{5}\right): \mathrm{m}\left(\mathrm{Co}(\mathrm{CO})_{8}\right)=7: 1$

(c) $\mathrm{m}\left(\mathrm{Fe}(\mathrm{CO})_{5}\right): \mathrm{m}\left(\mathrm{Co}(\mathrm{CO})_{8}\right)=4: 1$

(d) $\mathrm{m}\left(\mathrm{Fe}(\mathrm{CO})_{5}\right): \mathrm{m}\left(\mathrm{Co}(\mathrm{CO})_{8}\right)=5: 1$

Fig.1 The morphology of Fe-Co double-particle magnetic fluid in the magnetic field

Fig. 3 show the transmission electron micrograph of the Fe-Co double nanoparticle powder and the shape of the particles is nearly spherical when mass ratio of $\mathrm{Fe}(\mathrm{CO})_{5}$ and $\mathrm{Co}_{2}(\mathrm{CO})_{8}$ is $5: 1$. It can be seen that the particles have an average size of about $14 \mathrm{~nm}$ which is higher than the value determined by XRD. The reason may come from the overlap of the nanoparticles during the TEM analysis. The nano-magnetic particles uniform covered surfactant in the Fe-Co double nanoparticle magnetic fluid and favorable distribution with most particle size is approximate $14 \mathrm{~nm}$. According to reference [20] the stability of the magnetic fluid was estimated. After resting for 3 days, the particle suspension percentage of the entire magnetic fluid sample is keeping above $98 \%$. And it means that the magnetic particles can be coated completely by the surfactant and dispersed in the carrier liquid homogeneously.
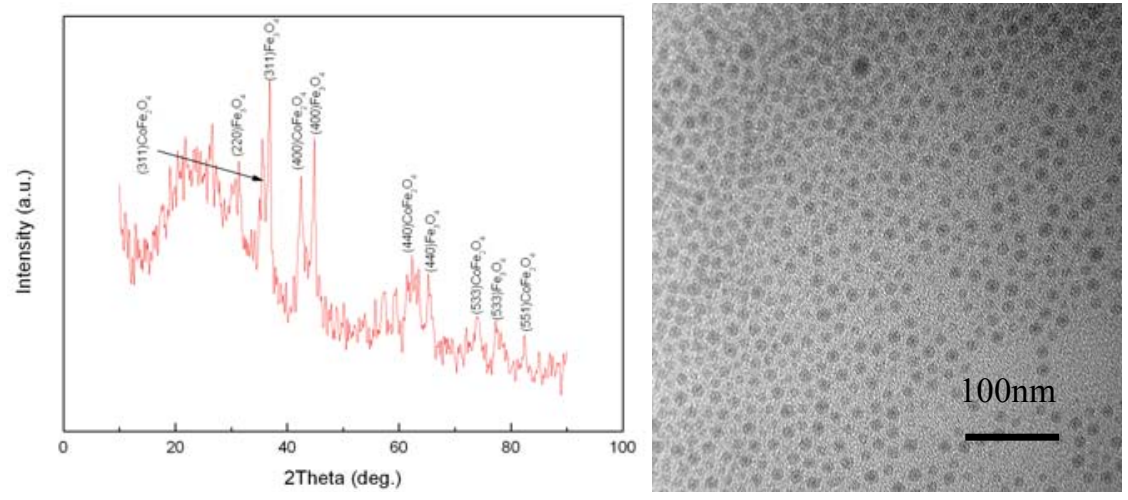

Fig. 2 X-ray diffraction pattern of Fe-Co double-particle

Fig.3. TEM image of Fe-Co double nanoparticle

magnetic fluid at the room temperature.

Fig. 4 shows the hysteresis loop of Fe-Co double-particle magnetic fluid in the magnetic field up to $5000 \mathrm{Gs}$ at room temperature when mass ratio of $\mathrm{Fe}(\mathrm{CO})_{5}$ and $\mathrm{Co}_{2}(\mathrm{CO})_{8}$ is $5: 1$. It is clear that the $\mathrm{Fe}-\mathrm{Co}$ double nanoparticle magnetic fluid is super paramagnetic functional materials with magnetic saturation of the $0.025 \mathrm{~T}$, namely the hysteresis loss, coercive force close to zero. In other words, it is reversible magnetization process, namely the hysteresis loss, coercive force close to zero, hysteresis loop is an origin of coordinates of S type curve, almost no hysteresis phenomenon, both of the double of the Fe-Co particles of magnetic liquid so there is no interaction between nanoparticles. High saturation magnetization (Ms) and chemical stability are the important indexes to value the performance of the magnetic liquid. This performance is mainly due to the characteristics of two magnetic liquid composed of magnetic particles and magnetic liquid volume fraction occupied by the decision. Co particles has strong ability of paramagnetic, after replacing Fe particles with part of Co particles, the paramagnetic ability of the magnetic liquid is greatly improved, and the liquid also shows the high saturation magnetization and good stability.

Fig. 5 show the dependence of the friction coefficient on friction time lubricated with the liquid paraffin, 10\# engine oil and $\mathrm{Fe}-\mathrm{Co}$ double-particle magnetic fluid when mass ratio of $\mathrm{Fe}(\mathrm{CO})_{5}$ and 
$\mathrm{Co}_{2}(\mathrm{CO})_{8}$ is 5:1. It is clear that the friction coefficient of all samples firstly gradually decrease then stabilization with increase friction time. Compared with the sample by conventional lubricant, the friction coefficient of Fe-Co double-particle magnetic fluid is notably lower.

Fig. 6 illustrates the friction coefficient of the Fe-Co double-particle magnetic fluid when mass ratio of $\mathrm{Fe}(\mathrm{CO})_{5}$ and $\mathrm{Co}_{2}(\mathrm{CO})_{8}$ is $5: 1$ and paraffin oil as a function of rotational speed. It is found that the decrease friction coefficient of all samples with increase of rotational speed. However, the friction coefficient of the Fe-Co double-particle magnetic fluid rapidly decreases than paraffin oil with increase of rotational speed. This indicates that the Fe-Co double-particle magnetic fluid better performance lubricating properties. It can be attributed to the $\mathrm{Fe}-\mathrm{Co}$ double-particle magnetic fluid may play as the rollers in the magnetic lubrications.

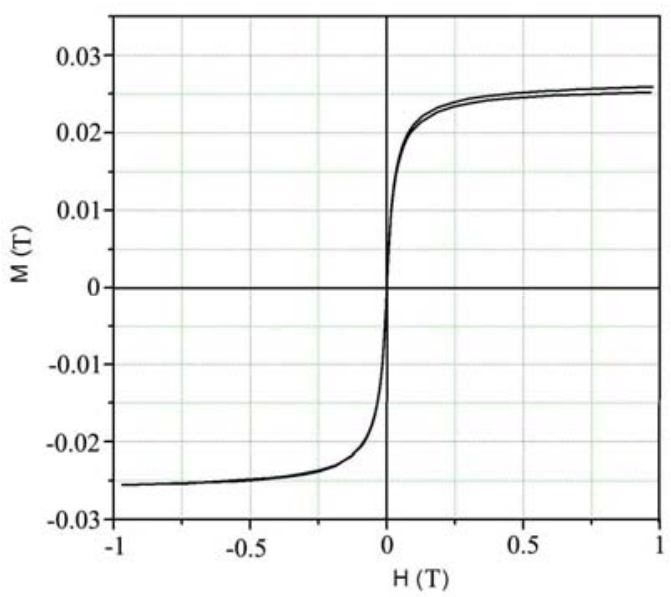

Fig.4. The hysteresis loops at room temperature of the Fe-Co double-particle magnetic fluid under the field.

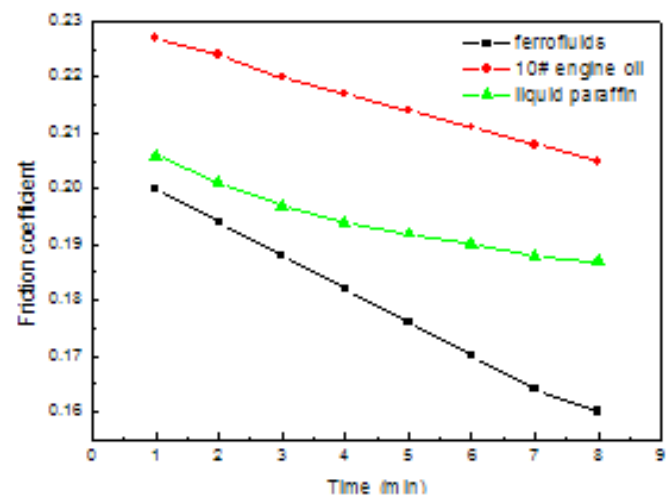

Fig.5. The dependence of the friction coefficient on friction time lubricated with the liquid paraffin, 10\# engine

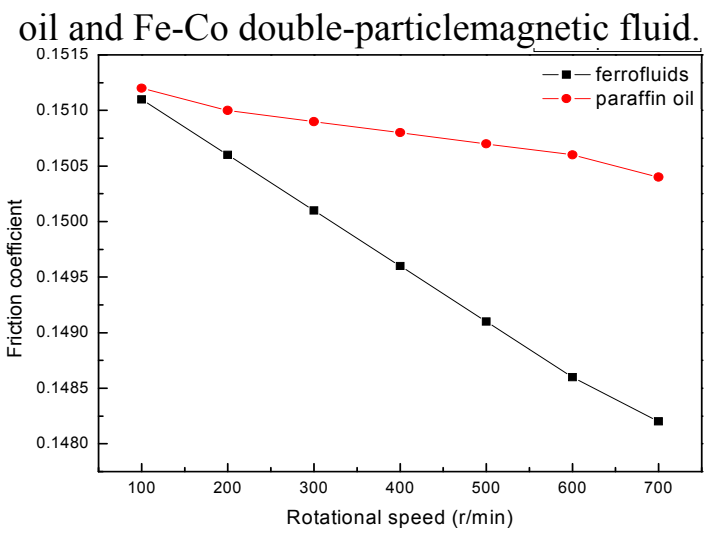

Fig.6. The dependence of the friction coefficient on rotational speed with the liquid paraffin oil and Fe-Co double-particle magnetic fluid.

As discussed above, the formation of Fe-Co double-particle magnetic fluid protective film with low hardness and elastic modulus on the worn surface can be considered as the main reason that the 
Fe-Co double-particle magnetic fluid exhibits excellent lubricating properties. It is clear that the higher the temperature is the more likely the melting of Fe-Co double-particle and soft film will be formed. Therefore, the friction of Fe-Co double-particle magnetic fluid is to reduce.

\section{Conclusions}

High saturation magnetization (Ms) and chemical stability are the important indexes to value the performance of the magnetic liquid. This performance is mainly due to the characteristics of two magnetic liquid composed of magnetic particles and magnetic liquid volume fraction occupied by the decision. Co particles has strong ability of paramagnetic, after replacing Fe particles with part of Co particles, the paramagnetic ability of the magnetic liquid is greatly improved, and the liquid also shows the high saturation magnetization and good stability. The morphology, structure and property of Fe-Co double nanoparticle magnetic fluid with the mass ratio of $\mathrm{Fe}(\mathrm{CO})_{5}$ and $\mathrm{Co}_{2}(\mathrm{CO})_{8}$ is $8: 1,7: 1$, 5:1,4:1 prepared by pulse laser deposition for the first time was systematically investigated. The results show that the peak height of Fe-Co double nanoparticle magnetic fluid under $5000 \mathrm{Gs}$ magnetic fluid gradually reduced with the increase mass ratio of $\mathrm{Fe}(\mathrm{CO})_{5}$ and $\mathrm{Co}_{2}(\mathrm{CO})_{8}$, and the peak height is biggest when mass ratio of $\mathrm{Fe}(\mathrm{CO})_{5}$ and $\mathrm{Co}_{2}(\mathrm{CO})_{8}$ is $5: 1$. It was indicated that the synthesized samples consisted of $\mathrm{Fe}_{3} \mathrm{O}_{4}$ and $\mathrm{CoFe}_{2} \mathrm{O}_{4}$ particles by $\mathrm{X}$-ray diffraction analysis. So the main content of the complex Fe-Co double nanoparticle magnetic fluid are $\mathrm{Fe}_{3} \mathrm{O}_{4}$ and $\mathrm{CoFe}_{2} \mathrm{O}_{4}$. The nano-magnetic particles uniform covered surfactant in the Fe-Co double nanoparticle magnetic fluid and favorable distribution with most particle size is approximate $14 \mathrm{~nm}$. At same time, Fe-Co double nanoparticle magnetic fluid is super paramagnetic functional materials with magnetic saturation of the $0.025 \mathrm{~T}$, namely the hysteresis loss, coercive force close to zero. The friction coefficient of the Fe-Co double-particle magnetic fluid rapidly decreases than paraffin oil with increase of rotational speed and friction time lubricated.

\section{Acknowledgements}

This study is supported by Program for Liaoning Excellent Talents in University (LJQ2013131), Natural Science Foundation of China (Grant No. 5401122), Shanghai Natural Science Foundation of Chian (Grant No. 13Zr1419400) and Supported by and Doctor Startup Foundation of Dalian University.

\section{References}

[1] J.L. Neuringer, R.E. Rosensweig, Phys. Fluids. 7 (1964)1927.

[2] B. Berkovsky, V. Bastovoi, Magnetic fluids and applications handbook, Begell House, New York, 1996.

[3] E. Blums, A. Cebers, and M. M. Maiorov, Magnetic fluids, Walter de Gruyter, Berlin ,1997

[4] M.V. Avdeev, V.L. Aksenov, M. Balasoiu, V.M. Garamus, A. Schreyer, Gy. Torok, L. Rosta, D. Bica,. L. Vekas. J. Coll. Interface Sci., 295(2006)100-107.

[5] M. Rasa, D. Bica, A.P. Philpse, L. Vekas .Eur. Phys. J. E, 7 (2002) 209-220.

[6] S. W. Charles. Rom. Rep. Phys..47(1995)249-264.

[7] A. O. Ivanov, O. B. Kuznetsova.Colloid J., 63(2001)60-67.

[8] V. Kuncser, G. Schinteie, B. Sahoo, W. Keune, D. Bica, L. Vékás, G. Filoti . J. Phys.: Cond. Matter, 19 (2007) 16.

[9] W. Huang, J.M. Wu, W. Guo, R. Li, L.Y. Cui. J.Magn. Magn. Mater., 307(2006):198-204.

[10] Maorun. Zhang. Journal of NingBo Uiversity. 11(1998):28-31.

[11] G.Z. Li. Journal of Hefei polytechnic university. 24(2001):248-250.

[12] Q. Wang, H. Yang. J Nanopart Res. 11(2009):1043-1051 
[13] R.Y. Hong, T.T. Pana, H.Z. Li. J. Magn. Magn. Mater., 303(2006):60-68.

[14] Maorun. Zhang, Z.C. Tao. Journal of Sichuan University. 36(2004):31-35.

[15] J. Kurfess, H.K. Muller. J. Magn. Magn. Mater., 85(1990):246-252.

[16] K. Nakatsuka, Y. Hama, J. Takahashi. J. Magn. Magn. Mater., 85(1990):207-209.

[17] J.W. Seo, S.J. Park. J. Magn. Magn. Mater., 192(1999):499-504.

[18]M.I. Piso. J. Magn. Magn. Mater., 201(1999):380-384.

[19] Anton I, Desata I, Vekas L. J. Magn. Magn. Mater., 65, 379 (1987).

[20] Nakatani I, Masayuki H, Kiyoshi O. J. Magn. Magn. Mater.,10(1993):122-124. 